\title{
On the Second Derivative of a Gaussian Process Envelope
}

\section{Ali Abdi}

Abstract-In this paper we explore some dynamic characteristics of the envelope of a bandpass Gaussian process, which are of interest in wireless fading channels. Specifically, we show that unlike the first derivative, the second derivative of the envelope, which appears in a number of applications, does not exist in the traditional mean square sense. However, we prove that the envelope is twice differentiable almost everywhere (with probability one), if the power spectrum of the bandpass Gaussian process satisfies a certain condition. We also derive an integral-form for the probability density function of the second derivative of the envelope, assuming an arbitrary power spectrum.

Index Terms-Envelope, Envelope second derivative, Gaussian process, Rayleigh process, Mean square differentiability, Almost everywhere differentiability, Differentiability with probability one, Fading channels.

List of figure captions:

Fig. 1. The joint probability density function of $R^{\prime \prime}$ and $\Theta^{\prime}$ for two different power spectra:

Upper: Exponential non-symmetric spectrum $w_{I}(f)=\exp \left(f-f_{m}\right),-2<f-f_{m}<2$,

Lower: Gaussian symmetric spectrum $w_{I}(f)=\exp \left[-\left(f-f_{m}\right)^{2}\right],-1<f-f_{m}<1$.

Fig. 2. The probability density function of $R^{\prime \prime}$ for two different power spectra:

Upper: Exponential non-symmetric spectrum $w_{I}(f)=\exp \left(f-f_{m}\right),-2<f-f_{m}<2$,

Lower: Gaussian symmetric spectrum $w_{I}(f)=\exp \left[-\left(f-f_{m}\right)^{2}\right], \quad-1<f-f_{m}<1$.

Fig. 3. The joint probability density function of $R$ and $R^{\prime \prime}$ for the Gaussian symmetric spectrum $w_{I}(f)=\exp \left[-\left(f-f_{m}\right)^{2}\right], \quad-1<f-f_{m}<1$. 


\section{INTRODUCTION}

The envelope of a bandpass Gaussian process is a slowly varying process that conveys useful information about the Gaussian process. For an arbitrary power spectrum, the envelope has a Rayleigh probability density function (PDF) [1], while it has been shown by several authors, independently, that its first derivative has a Gaussian PDF [2] [3] [4] [5] [6]. However, only the conditional PDF of the envelope second derivative has been studied so far, assuming an even-symmetric power spectrum [7]. On the other hand, in many real world cases such as wireless propagation channels where the scattering of waves might be nonisotropic, experimental results have shown that the underlying random process has a nonsymmetric power spectrum in general [8]. So, the assumption of having a power spectrum which is not necessarily even-symmetric is far from being of just intellectual or theoretical interest and we need to take into account this fact in deriving the PDF of the envelope second derivative.

The first derivative of the envelope can be shown to exist in the mean square (MS) sense. However, we prove that the second derivative does not exist in the MS sense. It seems that this fact has been overlooked in those studies [1] [7] [9] [10] [11] [12] where the second derivative of the envelope has been of concern. Obviously, until it can be demonstrated that the envelope is twice differentiable in some sense, it is meaningless to talk about its PDF and the associated statistical properties. In this paper we show that the second derivative of the envelope exists almost everywhere (AE). Using the equivalent terminology, the envelope is twice differentiable with probability one. This allows us to talk about the PDF of the envelope second derivative and its statistical characteristics.

The rest of the paper is organized as follows. In Section II we prove that the envelope is twice differentiable in the AE sense and not the MS sense. In order to derive the PDF of the envelope second derivative for the general case, i.e., an arbitrary power spectrum, we derive the joint PDF of the envelope and phase and their first two derivatives in Section III. Then we show that the PDF of the envelope second derivative can be expressed in terms of a single-fold integral, which cannot be solved analytically and needs to be computed numerically. The paper concludes with a summary given in Section IV. 


\section{SECOND-ORDER DifFERENTIABILITY OF THE ENVELOPE}

According to the Rice's representation [13] [14], a stationary, bandpass, and zero-mean Gaussian process $I(t)$ can be written as:

$I(t)=I_{c}(t) \cos 2 \pi f_{m} t-I_{s}(t) \sin 2 \pi f_{m} t$,

where $f_{m}$ is a representative midband frequency, and $I_{c}(t)$ and $I_{s}(t)$ are two joint stationary, lowpass, and zero-mean Gaussian processes. Using the polar representation we obtain:

$I(t)=R(t) \cos \left[2 \pi f_{m} t+\Theta(t)\right]$,

in which $R(t)$ and $\Theta(t)$ are the envelope and phase of $I(t)$, respectively, defined by:

$R(t)=\sqrt{I_{c}^{2}(t)+I_{s}^{2}(t)} \quad, \quad \tan \Theta(t)=I_{s}(t) / I_{c}(t)$.

In order to investigate the first- and the second-order MS differentiability of $R(t)$, we need the autocorrelation function of $R(t), \Re_{R}(\tau)=E[R(t) R(t+\tau)]$, given in p. 170 of [15]:

$\Re_{R}(\tau)=\frac{\pi b_{0}}{2}{ }_{2} F_{1}\left(\frac{-1}{2}, \frac{-1}{2} ; 1 ; \rho_{I}^{2}(\tau)\right)$,

where $b_{n}$ is the $n$th spectral moment of $I(t)$ :

$b_{n}=(2 \pi)^{n} \int_{0}^{\infty}\left(f-f_{m}\right)^{n} w_{I}(f) d f$,

and $w_{I}(f), f \geq 0$, is the one-sided power spectrum of $I(t)$, concentrated around $f_{m}$ and with a bandwidth much smaller than $f_{m}$. In Eq. (4), ${ }_{2} F_{1}(.,, ; . ;$.$) is the hypergeometric function [16],$ $\rho_{I}(\tau)=\sqrt{g^{2}(\tau)+h^{2}(\tau)} / b_{0}$, where $g(\tau)=E\left[I_{c}(t) I_{c}(t+\tau)\right]=E\left[I_{s}(t) I_{s}(t+\tau)\right]$ is the autocorrelation function of both $I_{c}(t)$ and $I_{s}(t)$ in (1), whereas $h(\tau)=E\left[I_{c}(t) I_{s}(t+\tau)\right]=-E\left[I_{s}(t) I_{c}(t+\tau)\right]$ is the crosscorrelation function between $I_{c}(t)$ and $I_{s}(t)$. According to [1], $g(\tau)$ and $h(\tau)$ can be expressed in terms of $w_{I}(f)$ :

$$
\begin{aligned}
& g(\tau)=\int_{0}^{\infty} w_{I}(f) \cos \left[2 \pi\left(f-f_{m}\right) \tau\right] d f, \\
& h(\tau)=\int_{0}^{\infty} w_{I}(f) \sin \left[2 \pi\left(f-f_{m}\right) \tau\right] d f .
\end{aligned}
$$

We assume that both $g(\tau)$ and $h(\tau)$ are differentiable up to order four.

Based on the following relations, obtained according to (5) and (6): 
$g(0)=b_{0}, \quad \dot{g}(0)=0, \quad \ddot{g}(0)=-b_{2}, \quad \dddot{g}(0)=0, \quad g^{(4)}(0)=b_{4}$,

$h(0)=0, \quad \dot{h}(0)=b_{1}, \quad \ddot{h}(0)=0, \quad \dddot{h}(0)=-b_{3}, \quad h^{(4)}(0)=0$,

it can be easily verified that:

$\rho_{I}(0)=1$,

$\dot{\rho}_{I}(0)=0$,

$\ddot{\rho}_{I}(0)=\frac{-b_{0} b_{2}+b_{1}^{2}}{b_{0}^{2}}$,

$\dddot{\rho}_{I}(0)=0$,

$\rho_{I}^{(4)}(0)=\frac{b_{4}}{b_{0}}-4 \frac{b_{1} b_{3}}{b_{0}^{2}}+6 \frac{b_{1}^{2} b_{2}}{b_{0}^{3}}-3 \frac{b_{1}^{4}}{b_{0}^{4}}$,

where dot denotes differentiation with respect to $\tau$. Now we list some properties of the hypergeometric function [16], which we need in the sequel:

${ }_{2} F_{1}\left(\frac{-1}{2}, \frac{-1}{2} ; 1 ; 1\right)={ }_{2} F_{1}\left(\frac{1}{2}, \frac{1}{2} ; 2 ; 1\right)=\frac{4}{\pi}$,

${ }_{2} F_{1}\left(\frac{3}{2}, \frac{3}{2} ; 3 ; 1\right)=\infty$

$\frac{d}{d z} \quad{ }_{2} F_{1}\left(\frac{-1}{2}, \frac{-1}{2} ; 1 ; z^{2}\right)=\frac{z}{2} \quad{ }_{2} F_{1}\left(\frac{1}{2}, \frac{1}{2} ; 2 ; z^{2}\right)$

$\frac{d^{2}}{d z^{2}} \quad{ }_{2} F_{1}\left(\frac{-1}{2}, \frac{-1}{2} ; 1 ; z^{2}\right)=\frac{1}{2} \quad\left[{ }_{2} F_{1}\left(\frac{1}{2}, \frac{1}{2} ; 2 ; z^{2}\right)+\frac{z^{2}}{4} \quad{ }_{2} F_{1}\left(\frac{3}{2}, \frac{3}{2} ; 3 ; z^{2}\right)\right]$.

Let $b_{0} \neq 0$, whereas $b_{1}$ up to $b_{4}$ are finite real numbers. Since $R(t)$ is a wide sense stationary process, it is MS differentiable if and only if $\mathfrak{R}_{R}(\tau)$ is twice differentiable at $\tau=0$ [13] [14] [17].

Based on (4), (8), and (9) we obtain:

$\ddot{\mathfrak{R}}_{R}(0)=-\frac{b_{0} b_{2}-b_{1}^{2}}{b_{0}}$,

which demonstrates the MS differentiability of $R(t)$ as $\left|\ddot{\mathfrak{R}}_{R}(0)\right|<\infty$. Similarly, $R(t)$ is twice MS differentiable if and only if the fourth derivative of $\mathfrak{R}_{R}(\tau)$ exists at $\tau=0$. However, according to (4), (8), and (9), we obtain the following relation:

$\mathfrak{R}_{R}^{(4)}(0)=\frac{\pi b_{0}}{2}\left[\left.3 \quad \ddot{\rho}_{I}^{2}(0) \frac{d^{2}}{d z^{2}}{ }_{2} F_{1}\left(\frac{-1}{2}, \frac{-1}{2} ; 1 ; z^{2}\right)\right|_{z=1}+\left.\rho_{I}^{(4)}(0) \quad \frac{d}{d z} \quad{ }_{2} F_{1}\left(\frac{-1}{2}, \frac{-1}{2} ; 1 ; z^{2}\right)\right|_{z=1}\right]$, 
which simplifies to $\mathfrak{R}_{R}^{(4)}(0)=\infty$. Hence, $R(t)$ is not MS differentiable, twice. Notice that the identity $\mathfrak{R}_{R^{\prime \prime}}(0)=\mathfrak{R}_{R}^{(4)}(0)$ [14] yields $E\left[R^{\prime \prime 2}\right]=\infty$. On the other hand, a simple expression is given in [7] for $E\left[R^{\prime \prime 2} \mid R\right]$, assuming an even-symmetric power spectrum. By averaging this result with respect to $R$, we obtain $E\left[R^{\prime \prime 2}\right]=\infty$ for an even-symmetric power spectrum, in agreement with our finding for the more general case of an arbitrary power spectrum.

So far, we have shown that the envelope is not twice MS differentiable. Nevertheless, in the following theorem, we prove that the envelope is twice AE differentiable:

Theorem: For the stationary, bandpass, and zero-mean Gaussian process $I(t)$ in (1), with the one-sided power spectrum $w_{I}(f)$, let:

$\int_{0}^{\infty}\left|f-f_{m}\right|^{4+\alpha} w_{I}(f) d f<\infty$

for some real $\alpha>0$. Then $R(t)$, the envelope of $I(t)$, is twice AE differentiable. In other words, $R^{\prime \prime}(t)$ exits almost everywhere (with probability one).

Proof: Let us define the lowpass-equivalent complex envelope of $I(t)$ as $\mathcal{I}(t)=I_{c}(t)+j I_{s}(t)=R(t) \exp [j \Theta(t)]$, where $j^{2}=-1$. Clearly, $\mathcal{I}(t)$ is a complex, stationary, lowpass, and zero-mean Gaussian process with the two-sided power spectrum $w_{\mathcal{I}}(f)=w_{I}\left(f+f_{m}\right)$, concentrated around $f=0$, and the autocorrelation function $\mathfrak{R}_{\mathcal{I}}(\tau)=\frac{1}{2} E\left[\mathcal{I}^{*}(t) \mathcal{I}(t+\tau)\right]=g(\tau)+j h(\tau)$, where $*$ denotes the complex conjugate ${ }^{1}$. Based on (6), it is easy to verify that $w_{\mathcal{I}}(f)$ is the Fourier transform of $\mathfrak{R}_{\mathcal{I}}(\tau)$. Using the new notation, the condition in (12) can be expressed in terms of $w_{\mathcal{I}}(f)$ as $\int_{-\infty}^{\infty}|f|^{4+\alpha} w_{\mathcal{I}}(f) d f<\infty$. Based on Theorem 5.2 in p. 46 of [17], $\mathfrak{R}_{\mathcal{I}^{\prime \prime}}(\tau)=\mathfrak{R}_{\mathcal{I}}^{(4)}(\tau)$ is a continuous function. Since $w_{\mathcal{I}^{\prime \prime}}(f)=(2 \pi f)^{4} w_{\mathcal{I}}(f)$ [14], (12) implies that $\int_{-\infty}^{\infty}|f|^{\alpha} w_{\mathcal{I}^{\prime \prime}}(f) d f<\infty$. Now, based on the continuity of $\Re_{\mathcal{I}^{\prime \prime}}(\tau)$ and the convergence of $\int_{-\infty}^{\infty}|f|^{\alpha} w_{\mathcal{I}^{\prime \prime}}(f) d f$ for some real $\alpha>0$ by hypothesis, Theorem 6.5 in p. 54 of [17] tells us that the sample functions of the complex, stationary, lowpass, and zero-mean Gaussian process $\mathcal{I}^{\prime \prime}(t)$ are continuous with probability one. ${ }^{2}$ In other words, $\mathcal{I}(t)$ is twice AE differentiable. This simply means

${ }^{1}$ In this paper, the autocorrelation function of a stationary real process $Z(t)$ is defined as $\Re_{Z}(\tau)=E[Z(t) Z(t+\tau)]$, whereas for a stationary complex process $\mathcal{Z}(t)$, the definition is $\mathfrak{R}_{\mathcal{Z}}(\tau)=\frac{1}{2} E\left[\mathcal{Z}^{*}(t) \mathcal{Z}(t+\tau)\right]$.

2 Strictly speaking, there exists a process equivalent to $\mathcal{I}^{\prime \prime}(t)$ which possesses continuous sample functions with probability one [17]. 
that its real and imaginary parts, $I_{c}(t)$ and $I_{s}(t)$, are twice AE differentiable as well. Notice that based on the following relation, derived from (3):

$R^{\prime}(t)=\frac{I_{c}(t) I_{c}^{\prime}(t)+I_{s}(t) I_{s}^{\prime}(t)}{R(t)}$,

$R^{\prime}(t)$ exists with probability one, as $I_{c}^{\prime}(t)$ and $I_{s}^{\prime}(t)$ exist with probability one.

To complete the proof, we take the derivative of (13) which yields:

$R^{\prime \prime}(t)=\frac{I_{c}(t) I_{c}^{\prime \prime}(t)+I_{s}(t) I_{s}^{\prime \prime}(t)+I_{c}^{\prime 2}(t)+I_{s}^{\prime 2}(t)-R^{\prime 2}(t)}{R(t)}$.

Since we have shown that all the derivatives in (14), i.e., $I_{c}^{\prime}(t), I_{s}^{\prime}(t), I_{c}^{\prime \prime}(t), I_{s}^{\prime \prime}(t)$, and $R^{\prime}(t)$ exist with probability one, we conclude that $R^{\prime \prime}(t)$ exists with probability one as well. In other words, $R(t)$ is twice AE differentiable. Q.E.D.

The instantaneous power, defined by $P(t)=R^{2}(t)$, is a process of interest in some applications [18] and inherits several properties of $R(t) .{ }^{3}$ According to [18], p. 60, the autocorrelation function of $P(t)$, defined by $\Re_{P}(\tau)=E[P(t) P(t+\tau)]$, can be written as

$\Re_{P}(\tau)=4 b_{0}^{2}+4\left[g^{2}(\tau)+h^{2}(\tau)\right]$

Since we have already assumed that both $g(\tau)$ and $h(\tau)$ are differentiable up to order four, the fourth derivative of $\mathfrak{R}_{P}(\tau)$ exists at $\tau=0$, which implies that $P(t)$ is twice MS differentiable. Therefore, in those applications where $P(t)$ can be used in place of $R(t)$, one can still employ the notion of MS differentiability.

We end this section with an interesting observation. The derivative of the phase in $(3), \Theta^{\prime}(t)$, is another process of interest in fading channels, as it represents the random frequency modulation (FM) of the fading signal in wireless channels [20]. Similar to $R^{\prime \prime}(t)$, it is proved in Theorem 9.3 in p. 73 of [17] that $\Theta^{\prime}(t)$ exists in the AE sense and not the MS sense. Other examples of such a process can be found in p. 537 of [21] and p. 67 of [22].

${ }^{3}$ For example, every maximum of $R(t)$ is a maximum of $P(t)$. This fact is used in [19] to calculate the expected number of maxima of $R(t)$, a problem which is easier to solve for $P(t)$. 


\section{III. $p_{R^{\prime \prime}}\left(r^{\prime \prime}\right)$ FOR AN ARbitrary Power SPECTRUM}

In this section, we concentrate on the PDF of the envelope second derivative, a process that we proved to exist in the AE sense, and not the traditional MS sense. For $t_{0}$, an arbitrary instant of time, the six dimensional random vector $\quad \mathbf{V}_{\text {rect }}=\left[\begin{array}{lllllll}I_{c} & I_{s}^{\prime} & I_{c}^{\prime \prime} & I_{s} & I_{c}^{\prime} & I_{s}^{\prime \prime}\end{array}\right]^{T}$, with $I_{c} \equiv I_{c}\left(t_{0}\right), \quad I_{s}^{\prime} \equiv I_{s}^{\prime}\left(t_{0}\right), \quad I_{c}^{\prime \prime} \equiv I_{c}^{\prime \prime}\left(t_{0}\right), \quad I_{s} \equiv I_{s}\left(t_{0}\right), \quad I_{c}^{\prime} \equiv I_{c}^{\prime}\left(t_{0}\right), \quad I_{s}^{\prime \prime} \equiv I_{s}^{\prime \prime}\left(t_{0}\right), \quad$ and $T$ as the transpose operator, is a Gaussian vector with zero mean-vector and the following covariance matrix [1]:

$$
\mathbf{M}=\left[\begin{array}{cccccc}
b_{0} & b_{1} & -b_{2} & 0 & 0 & 0 \\
b_{1} & b_{2} & -b_{3} & 0 & 0 & 0 \\
-b_{2} & -b_{3} & b_{4} & 0 & 0 & 0 \\
0 & 0 & 0 & b_{0} & -b_{1} & -b_{2} \\
0 & 0 & 0 & -b_{1} & b_{2} & b_{3} \\
0 & 0 & 0 & -b_{2} & b_{3} & b_{4}
\end{array}\right] .
$$

The PDF of $\mathbf{V}_{\text {rect }}$ can be written as:

$$
p_{\mathbf{v}_{\text {reet }}}\left(\mathbf{v}_{\text {rect }}\right)=\frac{1}{8 \pi^{3} B} \exp \left(\frac{-1}{2} \mathbf{v}_{\text {rect }}^{T} \mathbf{M}^{-1} \mathbf{v}_{\text {rect }}\right),
$$

where $\mathbf{M}^{-1}$ is the inverse of $\mathbf{M}$. The adjoint of $\mathbf{M}$, denoted by $\operatorname{adj}(\mathbf{M})$, is given by [1]:

$$
\operatorname{adj}(\mathbf{M})=\left[\begin{array}{cccccc}
B_{0} & B_{1} & -B_{2} & 0 & 0 & 0 \\
B_{1} & B_{22} & -B_{3} & 0 & 0 & 0 \\
-B_{2} & -B_{3} & B_{4} & 0 & 0 & 0 \\
0 & 0 & 0 & B_{0} & -B_{1} & -B_{2} \\
0 & 0 & 0 & -B_{1} & B_{22} & B_{3} \\
0 & 0 & 0 & -B_{2} & B_{3} & B_{4}
\end{array}\right],
$$

where:

$$
\begin{aligned}
& B=b_{0} b_{2} b_{4}+2 b_{1} b_{2} b_{3}-b_{2}{ }^{3}-b_{0} b_{3}{ }^{2}-b_{1}{ }^{2} b_{4}=\sqrt{\operatorname{det}(\mathbf{M})}, \\
& B_{0}=\left(b_{2} b_{4}-b_{3}^{2}\right) B \\
& B_{1}=-\left(b_{1} b_{4}-b_{2} b_{3}\right) B \\
& B_{2}=\left(b_{1} b_{3}-b_{2}^{2}\right) B \\
& B_{22}=\left(b_{0} b_{4}-b_{2}^{2}\right) B \\
& B_{3}=-\left(b_{0} b_{3}-b_{1} b_{2}\right) B \\
& B_{4}=\left(b_{0} b_{2}-b_{1}^{2}\right) B
\end{aligned}
$$


and $\operatorname{det}(\mathbf{M})$ is the determinant of $\mathbf{M}$. Clearly, $\operatorname{adj}(\mathbf{M})=\operatorname{det}(\mathbf{M}) \mathbf{M}^{-1}$. Since the elements of $\mathbf{V}_{\text {rect }}$ are linearly independent, $\operatorname{det}(\mathbf{M})>0$ [14]. Similarly, $B>0$ as $B$ is the determinant of the covariance matrix of three linearly independent random variables $I_{c}, I_{s}^{\prime}$, and $I_{c}^{\prime \prime}$. Moreover, using the Schwarz inequality [14], it can be easily verified that $B_{0}, B_{22}$, and $B_{4}$ are also positive.

According to (3), it can be easily shown that [1]:

$I_{c}=R \cos \Theta$,

$I_{s}^{\prime}=R^{\prime} \sin \Theta+R \Theta^{\prime} \cos \Theta$,

$I_{c}^{\prime \prime}=R^{\prime \prime} \cos \Theta-2 R^{\prime} \Theta^{\prime} \sin \Theta-R \Theta^{\prime 2} \cos \Theta-R \Theta^{\prime \prime} \sin \Theta$,

$I_{s}=R \sin \Theta$,

$I_{c}^{\prime}=R^{\prime} \cos \Theta-R \Theta^{\prime} \sin \Theta$,

$I_{s}^{\prime \prime}=R^{\prime \prime} \sin \Theta+2 R^{\prime} \Theta^{\prime} \cos \Theta-R \Theta^{\prime 2} \sin \Theta+R \Theta^{\prime \prime} \cos \Theta$,

where we have the six dimensional random vector $\mathbf{V}_{\text {polar }}=\left[\begin{array}{lllllll}R & R^{\prime} & R^{\prime \prime} & \Theta & \Theta^{\prime} & \Theta^{\prime \prime}\end{array}\right]^{T}$, with $R \equiv R\left(t_{0}\right), \quad R^{\prime} \equiv R^{\prime}\left(t_{0}\right), \quad R^{\prime \prime} \equiv R^{\prime \prime}\left(t_{0}\right), \quad \Theta \equiv \Theta\left(t_{0}\right), \quad \Theta^{\prime} \equiv \Theta^{\prime}\left(t_{0}\right), \quad$ and $\quad \Theta^{\prime \prime} \equiv \Theta^{\prime \prime}\left(t_{0}\right) . \quad$ The $\quad$ PDF $\quad$ of $\mathbf{V}_{\text {polar }}$ can be determined from the PDF of $\mathbf{V}_{\text {rect }}$ in (16) as:

$p_{\mathbf{v}_{\text {polar }}}\left(\mathbf{v}_{\text {polar }}\right)=\frac{p_{\mathbf{v}_{\text {rect }}}\left(\mathbf{v}_{\text {polar }}\right)}{\left|J\left(\mathbf{v}_{\text {polar }}\right)\right|}$,

in which $J($.$) is the Jacobian [14] of the transformation \mathbf{V}_{\text {rect }} \rightarrow \mathbf{V}_{\text {polar }}$ in (19):

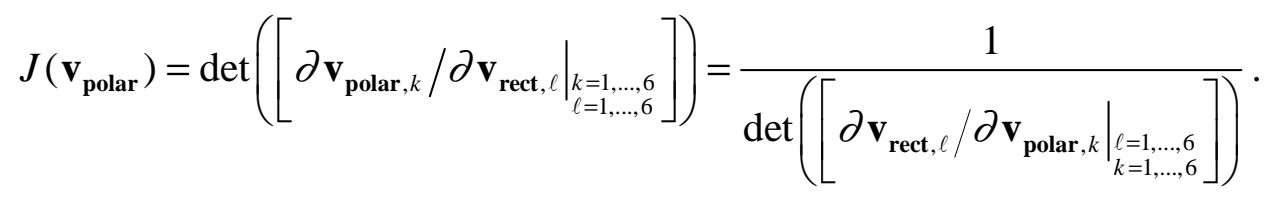

Based on (19), it can be shown that $J\left(\mathbf{v}_{\text {polar }}\right)=-1 / r^{3}$. So, by replacing $p_{\mathbf{v}_{\text {rect }}}$ (.) in (20) with (16), and after some algebraic manipulations, we obtain:

$p_{\mathbf{v}_{\text {polar }}}\left(\mathbf{v}_{\text {polar }}\right)=\frac{r^{3}}{8 \pi^{3} B} \exp \left(\frac{-1}{2 B^{2}} Q\left(\mathbf{v}_{\text {polar }}\right)\right)$,

where:

$$
\begin{aligned}
Q\left(\mathbf{v}_{\text {polar }}\right)=B_{0} r^{2} & +2 B_{1} r^{2} \theta^{\prime}-2 B_{2}\left(r r^{\prime \prime}-r^{2} \theta^{\prime 2}\right)+B_{22}\left(r^{\prime 2}+r^{2} \theta^{\prime 2}\right) \\
& -2 B_{3}\left(-2 r^{\prime 2} \theta^{\prime}+r r^{\prime \prime} \theta^{\prime}-r^{2} \theta^{\prime 3}-r r^{\prime} \theta^{\prime \prime}\right) \\
& +B_{4}\left(r^{\prime \prime 2}+4 r^{\prime 2} \theta^{\prime 2}-2 r r^{\prime \prime} \theta^{\prime 2}+r^{2} \theta^{\prime 4}+4 r r^{\prime} \theta^{\prime} \theta^{\prime \prime}+r^{2} \theta^{\prime \prime 2}\right) .
\end{aligned}
$$

Notice that $Q\left(\mathbf{v}_{\text {polar }}\right)$ is independent of $\theta$. Moreover, rearranging the terms in $Q\left(\mathbf{v}_{\text {polar }}\right)$ as: 


$$
\begin{aligned}
Q\left(\mathbf{v}_{\text {polar }}\right)=B_{0} r^{2} & +2 B_{1} r^{2} \theta^{\prime}-2 B_{2}\left(r r^{\prime \prime}-r^{2} \theta^{\prime 2}\right)+B_{22} r^{2} \theta^{\prime 2} \\
& -2 B_{3}\left(r r^{\prime \prime} \theta^{\prime}-r^{2} \theta^{\prime 3}\right)+B_{4}\left(r^{\prime \prime 2}-2 r r^{\prime \prime} \theta^{\prime 2}+r^{2} \theta^{\prime 4}\right) \\
& +\left(B_{22}+4 B_{3} \theta^{\prime}+4 B_{4} \theta^{\prime 2}\right) r^{\prime 2}+2 r r^{\prime}\left(B_{3}+2 B_{4} \theta^{\prime}\right) \theta^{\prime \prime}+B_{4} r^{2} \theta^{\prime \prime 2},
\end{aligned}
$$

reveals that as well as $\theta$, integration of $p_{\mathbf{v}_{\text {polar }}}\left(\mathbf{v}_{\text {polar }}\right)$ with respect to $\theta^{\prime \prime}$ can be done easily according to the following result in p. 307 of [23]:

$$
\int_{-\infty}^{\infty} \exp \left(-\mu z^{2}-v z\right) d z=\sqrt{\frac{\pi}{\mu}} \exp \left(\frac{v^{2}}{4 \mu}\right), \quad \mu>0 .
$$

Note that in our case $\mu=B_{4} r^{2} /\left(2 B^{2}\right)$, which is positive. Hence, integration of $p_{\mathbf{v}_{\text {polar }}}\left(\mathbf{v}_{\text {polar }}\right)$ with respect to $\theta$ and $\theta^{\prime \prime}$ gives the following result:

$$
\begin{aligned}
p_{R R^{\prime} R^{\prime \prime} \Theta^{\prime}}\left(r, r^{\prime}, r^{\prime \prime}, \theta^{\prime}\right)= & \int_{\theta^{\prime \prime}=-\infty}^{\infty} \int_{\theta=0}^{2 \pi} p_{\mathbf{v}_{\text {polar }}}\left(\mathbf{v}_{\text {polar }}\right) d \theta d \theta^{\prime \prime} \\
& =2 \pi \times \frac{B}{r} \sqrt{\frac{2 \pi}{B_{4}}} \exp \left(\frac{1}{2 B^{2}}\left\{\frac{B_{3}^{2}}{B_{4}}+4 B_{3} \theta^{\prime}+4 B_{4} \theta^{\prime 2}\right\} r^{\prime 2}\right) \\
& \times \frac{r^{3}}{8 \pi^{3} B} \exp \left(\frac{-1}{2 B^{2}}\left\{\begin{array}{l}
B_{0} r^{2}+2 B_{1} r^{2} \theta^{\prime}-2 B_{2}\left(r r^{\prime \prime}-r^{2} \theta^{\prime 2}\right)+B_{22} r^{2} \theta^{\prime 2} \\
-2 B_{3}\left(r r^{\prime \prime} \theta^{\prime}-r^{2} \theta^{\prime 3}\right)+B_{4}\left(r^{\prime \prime 2}-2 r r^{\prime \prime} \theta^{\prime 2}+r^{2} \theta^{\prime 4}\right) \\
+\left(B_{22}+4 B_{3} \theta^{\prime}+4 B_{4} \theta^{\prime 2}\right) r^{\prime 2}
\end{array}\right)\right) .
\end{aligned}
$$

Simplification of the above result yields:

$$
p_{R R^{\prime} R^{\prime \prime} \Theta^{\prime}}\left(r, r^{\prime}, r^{\prime \prime}, \theta^{\prime}\right)=p_{R R^{\prime \prime} \Theta^{\prime}}\left(r, r^{\prime \prime}, \theta^{\prime}\right) p_{R^{\prime}}\left(r^{\prime}\right)
$$

where:

$$
\begin{aligned}
& p_{R R^{\prime \prime} \Theta^{\prime}}\left(r, r^{\prime \prime}, \theta^{\prime}\right)=\frac{r^{2}}{2 \pi \sqrt{b_{0} B}} \exp \left(\frac{-1}{2 B^{2}}\left\{\begin{array}{l}
B_{0} r^{2}+2 B_{1} r^{2} \theta^{\prime}-2 B_{2}\left(r r^{\prime \prime}-r^{2} \theta^{\prime 2}\right)+B_{22} r^{2} \theta^{\prime 2} \\
-2 B_{3}\left(r r^{\prime \prime} \theta^{\prime}-r^{2} \theta^{\prime 3}\right)+B_{4}\left(r^{\prime \prime 2}-2 r r^{\prime \prime} \theta^{\prime 2}+r^{2} \theta^{\prime 4}\right)
\end{array}\right\}\right), \\
& p_{R^{\prime}}\left(r^{\prime}\right)=\frac{1}{\sqrt{2 \pi \sigma_{R^{\prime}}^{2}}} \exp \left(\frac{-r^{\prime 2}}{2 \sigma_{R^{\prime}}^{2}}\right),
\end{aligned}
$$

and:

$$
\sigma_{R^{\prime}}^{2}=\frac{B^{2} B_{4}}{B_{22} B_{4}-B_{3}^{2}}=\frac{b_{0} b_{2}-b_{1}^{2}}{b_{0}}=\frac{B_{4}}{b_{0} B}
$$

To check the validity of (27)-(30), we consider some known results. Integration of (28) with respect to $r^{\prime \prime}$ and $\theta^{\prime}$ yields $p_{R}(r)=r \exp \left(-r^{2} / 2 b_{0}\right) / b_{0}$ [1], while after integrating (28) with respect to $r$ and $r^{\prime \prime}$ 
we obtain $p_{\Theta^{\prime}}\left(\theta^{\prime}\right)=\left(b_{0} b_{2}-b_{1}^{2}\right)\left(b_{0} \theta^{\prime 2}-2 b_{1} \theta^{\prime}+b_{2}\right)^{-3 / 2} /\left(2 \sqrt{b_{0}}\right)$ [9]. It is easy to verify $E\left[R^{2}\right]=2 b_{0}$ using the expression given for $p_{R}(r)$, which is equal to $\mathfrak{R}_{R}(0)$, calculated according to (4) and (9). Also notice that the identity $\mathfrak{R}_{R^{\prime}}(0)=-\ddot{\mathfrak{X}}_{R}(0)$ [14], in conjunction with (10), results in $\sigma_{R^{\prime}}^{2}=\left(b_{0} b_{2}-b_{1}^{2}\right) / b_{0}$, in complete agreement with (30).

As can be deduced from (27) and (29), $R^{\prime}$ is a Gaussian random variable, independent of $R$ and $R^{\prime \prime}$. The Gaussianity of $R^{\prime}$ and also its independence of $R$ have been reported in [2] [4] [5] [6] for an arbitrary $w_{I}(f)$, while for a symmetric $w_{I}(f)$ about $f_{m}$, these properties are given in [7] and p. 75 of [24]. It may be worth mentioning that although the random variable $R^{\prime}$ is independent of $R$ and $R^{\prime \prime}$, it does not necessarily mean that the random process $R^{\prime}(t)$ is independent of $R(t)$ and $R^{\prime \prime}(t)$. For example, as suggested by one of the referees, it can be easily shown that $R^{\prime}(t)$ and $R(t)$ are dependent random processes. In fact, the crosscorrelation function between $R(t)$ and $R^{\prime}(t)$, defined by $\mathfrak{R}_{R R^{\prime}}(\tau)=E\left[R(t) R^{\prime}(t+\tau)\right]$, is given by $\mathfrak{R}_{R R^{\prime}}(\tau)=\dot{\mathfrak{R}}_{R}(\tau)$ [14], with $\mathfrak{R}_{R}(\tau)$ given in (4). Clearly, $E\left[R(t) R^{\prime}(t+\tau)\right]$ is not identically zero, whereas $E\left[R^{\prime}(t+\tau)\right]=0$ for all $t$ and $\tau$, according to (29). Therefore, $E\left[R(t) R^{\prime}(t+\tau)\right] \neq E[R(t)] E\left[R^{\prime}(t+\tau)\right]$, which implies that $R(t)$ and $R^{\prime}(t)$ are dependent random processes. Another example of this sort can be found in p. 170 of [15].

To obtain $p_{R^{\prime \prime}}\left(r^{\prime \prime}\right)$, first we need to derive a closed-form expression for either $p_{R R^{\prime \prime}}\left(r, r^{\prime \prime}\right)$ or $p_{R^{\prime \prime} \Theta^{\prime}}\left(r^{\prime \prime}, \theta^{\prime}\right)$. The bivariate PDF $p_{R R^{\prime \prime}}\left(r, r^{\prime \prime}\right)$ can be written in terms of $p_{R R^{\prime \prime} \Theta^{\prime}}\left(r, r^{\prime \prime}, \theta^{\prime}\right)$ in $(28)$ as:

$p_{R R^{\prime \prime}}\left(r, r^{\prime \prime}\right)=\frac{r^{2}}{2 \pi \sqrt{b_{0} B}} \exp \left(\frac{B_{0} r^{2}-2 B_{2} r r^{\prime \prime}+B_{4} r^{\prime \prime 2}}{-2 B^{2}}\right) \int_{\theta^{\prime}=-\infty}^{\infty} \exp \left(\frac{-1}{2 B^{2}} \sum_{i=1}^{4} \delta_{i} \theta^{\prime i}\right) d \theta^{\prime}$,

where:

$\delta_{1}=2\left(B_{1} r-B_{3} r^{\prime \prime}\right) r$

$\delta_{2}=\left[\left(B_{22}+2 B_{2}\right) r-2 B_{4} r^{\prime \prime}\right] r$,

$\delta_{3}=2 B_{3} r^{2}$,

$\delta_{4}=B_{4} r^{2}$.

The integral in (31) has not been addressed in [23] and seems that cannot be written in terms of known mathematical functions. However, in Appendix A we derive a closed-from expression for $p_{R R^{\prime \prime}}\left(r, r^{\prime \prime}\right)$, assuming an even-symmetric power spectrum. On the other hand, the bivariate PDF $p_{R^{\prime \prime} \Theta^{\prime}}\left(r^{\prime \prime}, \theta^{\prime}\right)$ can be expressed in terms of $p_{R R^{\prime \prime} \Theta^{\prime}}\left(r, r^{\prime \prime}, \theta^{\prime}\right)$ in (28) as: 
$p_{R^{\prime \prime} \Theta^{\prime}}\left(r^{\prime \prime}, \theta^{\prime}\right)=\frac{1}{2 \pi \sqrt{b_{0} B}} \exp \left(\frac{B_{4} r^{\prime \prime 2}}{-2 B^{2}}\right) \int_{r=0}^{\infty} r^{2} \exp \left(\frac{-1}{2 B^{2}} \sum_{i=1}^{2} \lambda_{i} r^{i}\right) d r$

in which:

$\lambda_{1}=-2\left(B_{2}+B_{3} \theta^{\prime}+B_{4} \theta^{\prime 2}\right) r^{\prime \prime}$,

$\lambda_{2}=B_{0}+2 B_{1} \theta^{\prime}+\left(2 B_{2}+B_{22}\right) \theta^{\prime 2}+2 B_{3} \theta^{\prime 3}+B_{4} \theta^{\prime 4}$.

Since the covariance matrix $\mathbf{M}$ in (15) is positive definite, its adjoint, given in (17) is positive definite as well. This implies that for any nonzero vector $\mathbf{x}$, we have $\mathbf{x}^{T} \operatorname{adj}(\mathbf{M}) \mathbf{x}>0$. The particular vector $\mathbf{x}=\left[\begin{array}{llllll}-1 & -\theta^{\prime} & \theta^{\prime 2} & 0 & 0 & 0\end{array}\right]^{T}$ yields $\mathbf{x}^{T} \operatorname{adj}(\mathbf{M}) \mathbf{x}=\lambda_{2}>0$, which holds for any $\theta^{\prime}$. On the other hand, we have the following result given in p. 338 of [23]:

$$
\int_{0}^{\infty} z^{2} \exp \left(-\mu z^{2}-2 v z\right) d z=\frac{-v}{2 \mu^{2}}+\sqrt{\frac{\pi}{\mu^{5}}} \frac{2 v^{2}+\mu}{4} \exp \left(\frac{v^{2}}{\mu}\right) \operatorname{erfc}\left(\frac{v}{\sqrt{\mu}}\right), \quad \mu>0,
$$

where:

$\operatorname{erfc}(z)=1-\frac{2}{\sqrt{\pi}} \int_{0}^{z} \exp \left(-s^{2}\right) d s$.

Notice that the convergence condition $\mu>0$ in (35) corresponds to $\lambda_{2}>0$ in (33). Therefore, based on (35), the bivariate PDF $p_{R^{\prime \prime} \Theta^{\prime}}\left(r^{\prime \prime}, \theta^{\prime}\right)$ can be obtained by simplifying (33) to:

$$
p_{R^{\prime \prime} \Theta^{\prime}}\left(r^{\prime \prime}, \theta^{\prime}\right)=\frac{-B^{3 / 2} \lambda_{1}}{4 \pi b_{0}^{1 / 2} \lambda_{2}^{2}} \exp \left(\frac{B_{4} r^{\prime \prime 2}}{-2 B^{2}}\right)+\frac{B^{5 / 2}}{8^{1 / 2} \pi^{1 / 2} b_{0}^{1 / 2} \lambda_{2}^{3 / 2}}\left(1+\frac{\lambda_{1}^{2}}{4 B^{2} \lambda_{2}}\right) \operatorname{erfc}\left(\sqrt{\frac{\lambda_{1}^{2}}{8 B^{2} \lambda_{2}}}\right) \exp \left(\frac{\lambda r^{\prime \prime 2}}{-2 B^{2} \lambda_{2}}\right),
$$

where:

$\lambda=B_{0} B_{4}-B_{2}^{2}+2\left(B_{1} B_{4}-B_{2} B_{3}\right) \theta^{\prime}+\left(B_{22} B_{4}-B_{3}^{2}\right) \theta^{\prime 2}$.

Note that the discriminant of the quadratic equation $\lambda\left(\theta^{\prime}\right) \equiv 0$ can be shown to be $-4 B_{4} \sqrt{\operatorname{det}(\operatorname{adj}(\mathbf{M}))}<0$, which along with $B_{22} B_{4}-B_{3}^{2}=b_{0} B^{3}>0$, proves that $\lambda>0$ for any $\theta^{\prime}$. We have already shown the same property for $\lambda_{2}$. The two properties $\lambda>0$ and $\lambda_{2}>0$ assure that the second exponential function in (36) does not blow up as $\left|r^{\prime \prime}\right|$ increases. In Fig. 1 we have plotted $p_{R^{\prime \prime} \Theta^{\prime}}\left(r^{\prime \prime}, \theta^{\prime}\right)$ for two different power spectra: the exponential non-symmetric spectrum $w_{I}(f)=\exp \left(f-f_{m}\right),-2<f-f_{m}<2$, and the Gaussian symmetric spectrum $w_{I}(f)=\exp \left[-\left(f-f_{m}\right)^{2}\right],-1<f-f_{m}<1$. Apparently, it is not possible to derive a closed-form 
solution for $p_{R^{\prime \prime}}\left(r^{\prime \prime}\right)$ by integrating $p_{R^{\prime \prime} \Theta^{\prime}}\left(r^{\prime \prime}, \theta^{\prime}\right)$ in (36), assuming an arbitrary power spectrum. Hence we leave it as

$p_{R^{\prime \prime}}\left(r^{\prime \prime}\right)=\int_{\theta^{\prime}=-\infty}^{\infty} p_{R^{\prime \prime} \Theta^{\prime}}\left(r^{\prime \prime}, \theta^{\prime}\right) d \theta^{\prime}$

In Fig. 2 we have plotted $p_{R^{\prime \prime}}\left(r^{\prime \prime}\right)$ for the above exponential and Gaussian spectra.

It may be argued that based on the following representations for $R^{\prime \prime}$, a closed-form expression may be obtained for $p_{R^{\prime \prime}}\left(r^{\prime \prime}\right)$ :

$R^{\prime \prime}=\lim _{h \rightarrow 0} \frac{R\left(t_{0}-h\right)-2 R+R\left(t_{0}+h\right)}{h^{2}}$,

$R^{\prime \prime}=\lim _{h \rightarrow 0} \frac{R^{\prime}\left(t_{0}+h\right)-R^{\prime}}{h}$.

The multivariate joint PDF of $R(t)$ is given in [25] and [26], in two different complicated forms (see also Theorem 6 in p. 37 of [27]). For the trivariate PDF, which we need in (38), the reader may refer to p. 92 of [17], p. 67 of [28], or [29] (see also [30]). However, deriving an expression for $p_{R^{\prime \prime}}\left(r^{\prime \prime}\right)$, based on the representation given in (38), seems to be very hard. On the other hand, a quick look at [31] immediately reveals that even for a symmetric power spectrum, the bivariate $\operatorname{PDF}$ of $R^{\prime}(t)$ is so complicated that makes (39) useless for our purpose. So, neither (38) nor (39) help us in finding a compact form for $p_{R^{\prime \prime}}\left(r^{\prime \prime}\right)$ in terms of tabulated functions.

\section{Conclusion}

In this paper and for a bandpass Gaussian process with any power spectrum, we have shown that the envelope second derivative does not exist in the mean square sense. However, we have proved that the envelope is twice differentiable almost everywhere (with probability one), provided that the power spectrum of the Gaussian process meets a certain condition. We have also derived an integral-form for the probability density function of the envelope second derivative. One immediate application of the results of this paper is the characterization of the dynamic behavior of multipath fading channels [32] [33], where the derivatives of the envelope and the associated probability density functions are of concern. 
APPENDIX A

\section{$p_{R R^{\prime \prime}}\left(r, r^{\prime \prime}\right)$ FOR AN EVEn-SYMMETRIC POWER SPECTRUM [34]}

When $w_{I}(f)$ has even symmetry about $f_{m}$, we obtain $b_{1}=b_{3}=0$ and then $B_{1}=B_{3}=0$. For this particular case, the integral in (31) can be expressed in terms of the parabolic cylinder function, available in standard mathematical softwares such as Mathematica@.

For a symmetric $w_{I}(f)$ we have $\delta_{1}=\delta_{3}=0$ in (32). According to the following relation in $\mathrm{p}$. 337 of [23]:

$\int_{0}^{\infty} z^{\kappa-1} \exp \left(-\rho z^{2}-\tau z\right) d z=(2 \rho)^{-\kappa / 2} \Gamma(\kappa) \exp \left(\frac{\tau^{2}}{8 \rho}\right) D_{-\kappa}\left(\frac{\tau}{\sqrt{2 \rho}}\right), \quad \rho>0, \kappa>0$,

and after the simple change of variable $z=\zeta^{2}$ we obtain:

$\int_{0}^{\infty} \zeta^{2 \kappa-1} \exp \left(-\rho \zeta^{4}-\tau \zeta^{2}\right) d \zeta=\frac{1}{2}(2 \rho)^{-\kappa / 2} \Gamma(\kappa) \exp \left(\frac{\tau^{2}}{8 \rho}\right) D_{-\kappa}\left(\frac{\tau}{\sqrt{2 \rho}}\right), \quad \rho>0, \kappa>0$,

where $\Gamma($.$) is the gamma function and D_{-\kappa}($.$) is the parabolic cylinder function of order -\kappa$. For $\kappa=1 / 2$, the above integral reduces to Eq. (15) of [10], if we express $D_{-1 / 2}($.$) in terms of the modified$

Bessel functions of the first and the second kind. However, application of the parabolic cylinder function results in a more compact expression. Using (A.2), integration with respect to $\theta^{\prime}$ in (31) can be easily carried out:

$$
p_{R R^{\prime \prime}}\left(r, r^{\prime \prime}\right)=\frac{r^{3 / 2}}{2 \sqrt{\pi} \sqrt{b_{0}} B_{4}^{1 / 4}} \exp \left(\frac{B_{0} r^{2}-2 B_{2} r r^{\prime \prime}+B_{4} r^{\prime \prime 2}}{-2 B^{2}}\right) \exp \left(\frac{\delta_{2}^{2}}{16 B^{2} \delta_{4}}\right) D_{-\frac{1}{2}}\left(\frac{\delta_{2}}{2 B \sqrt{\delta_{4}}}\right) .
$$

After replacing $B, \quad B_{0}, \quad B_{2}, \quad B_{22}$, and $B_{4}$ with $b_{n}$ 's according to (18) and also replacing $\delta_{2}$ and $\delta_{4}$ by their definitions in (32), we finally obtain:

$$
\begin{aligned}
p_{R R^{\prime}}\left(r, r^{\prime \prime}\right)= & \frac{r^{3 / 2}}{2 \pi^{1 / 2} b_{0}^{3 / 4} b_{2}^{1 / 2} c^{1 / 4}} \exp \left(\frac{-1}{2 c}\left\{\frac{b_{0}}{2} r^{\prime \prime 2}+b_{2}(2-b) r r^{\prime \prime}+\frac{2 b_{0} b_{4}-b^{2} b_{2}^{2}}{2 b_{0}} r^{2}\right\}\right) \\
& \times D_{-\frac{1}{2}}\left(\frac{-b b_{2}}{\sqrt{b_{0} c}} r-\sqrt{\frac{b_{0}}{c}} r^{\prime \prime}\right),
\end{aligned}
$$

where: 
$b=\frac{1}{2}\left(3-\frac{b_{0} b_{4}}{b_{2}{ }^{2}}\right)$,

$c=b_{0} b_{4}-b_{2}^{2}$.

Note that according to the Schwarz inequality $b<1$ and $c>0$. In Fig. 3 we have plotted $p_{R R^{\prime \prime}}\left(r, r^{\prime \prime}\right)$ for the Gaussian symmetric spectrum $w_{I}(f)=\exp \left[-\left(f-f_{m}\right)^{2}\right],-1<f-f_{m}<1$.

\section{ACKNOWLEDGEMENT}

The author would like to thank the referee for careful examination of the manuscript and insightful comments.

\section{REFERENCES}

[1] S. O. Rice, "Mathematical analysis of random noise," reprinted in Selected Papers on Noise and Stochastic Processes. N. Wax, Ed., New York: Dover, 1954, pp. 133-294.

[2] D. Middleton, "Spurious signals caused by noise in triggered circuits," J. Appl. Phys., vol. 19, pp. 817-830, 1948.

[3] W. M. Brown, "Some results on noise through circuits," IRE Trans. Inform. Theory, vol. 5, pp. 217-227, 1959.

[4] M. R. Leadbetter, "On crossings of levels and curves by a wide class of stochastic processes," Ann. Math. Statist., vol. 37, pp. 260-267, 1966.

[5] A. M. Hasofer, "The joint distribution of the envelope of a Gaussian process and its derivative," Austral. J. Statist., vol. 15, pp. 215-216, 1973.

[6] J. Komaili, L. A. Ferrari, and P. V. Sankar, "Estimating the bandwidth of a normal process from the level crossings of its envelope," IEEE Trans. Acoust., Speech, Signal Processing, vol. 35, pp. 1481-1483, 1987.

[7] B. N. Zvyaghintsev, "Some statistical properties of the second derivative of the envelope of a normal random process," Radio Eng. Electron. Phys., vol. 10, pp. 635-637, 1965.

[8] A. Abdi, J. A. Barger, and M. Kaveh, "A parametric model for the distribution of the angle of arrival and the associated correlation function and power spectrum at the mobile station," to appear in IEEE Trans. Vehic. Technol.. 
[9] S. O. Rice, "Statistical properties of a sine wave plus random noise," Bell Syst. Tech. J., vol. 27, pp. 109-157, 1948.

[10] N. M. Blachman, "The distributions of local extrema of Gaussian noise and of its envelope," IEEE Trans. Inform. Theory, vol. 45, pp. 2115-2121, 1999.

[11] R. Narasimhan and D. C. Cox, "Speed estimation in wireless systems using wavelets," IEEE Trans. Commun., vol. 47, pp. 1357-1364, 1999.

[12] R. Narasimhan and D. C. Cox, "Mean and variance of the local maxima of a Rayleigh fading envelope," IEEE Commun. Lett., vol. 4, pp. 352-353, 2000.

[13] W. A. Gardner, Introduction to Random Processes with Applications to Signals and Systems, 2nd ed., Singapore: McGraw-Hill, 1990.

[14] A. Papoulis, Probability, Random Variables, and Stochastic Processes, 3rd ed., Singapore: McGraw-Hill, 1991.

[15] W. B. Davenport, Jr. and W. L. Root, An Introduction to the Theory of Random Signals and Noise. New York: McGraw-Hill, 1958.

[16] W. Magnus, F. Oberhettinger, and R. P. Soni, Formulas and Theorems for the Special Functions of Mathematical Physics, 3rd ed., New York: Springer, 1966.

[17] K. S. Miller, Complex Stochastic Processes: An Introduction to Theory and Application. Reading, MA: Addison-Wesley, 1974.

[18] G. L. Stuber, Principles of Mobile Communication, 2nd ed., Boston, MA: Kluwer, 2001.

[19] A. Abdi and S. Nader-Esfahani, "Expected number of maxima in the envelope of a spherically invariant random process," revised version submitted to IEEE Trans. Inform. Theory.

[20] W. C. Jakes, Jr., Ed., Microwave Mobile Communications. New York: Wiley, 1974.

[21] J. L. Doob, Stochastic Processes. New York: Wiley, 1953.

[22] A. M. Yaglom, Correlation Theory of Stationary and Related Random Functions I: Basic Results. New York: Springer, 1987.

[23] I. S. Gradshteyn and I. M. Ryzhik, Table of Integrals, Series, and Products, corrected and enlarged ed., A. Jeffrey, Ed., New York: Academic, 1980.

[24] N. M. Blachman, Noise and Its Effect on Communication, 2nd ed., Malabar, FL: Krieger, 1982. 
[25] W. C. Hoffman, "The joint distribution of $n$ successive outputs of a linear detector," J. Appl. Phys., vol. 25, pp. 1006-1007, 1954.

[26] K. S. Miller and R. I. Bernstein, "An analysis of coherent integration and its application to signal detection," IRE Trans. Inform. Theory, vol. 3, pp. 237-248, 1957.

[27] K. S. Miller, Multivariate Distributions. Huntington, NY: Krieger, 1975.

[28] V. I. Tikhonov and A. A. Tolkachev, "The effect of non-normal fluctuations on linear systems," in Non-Linear Transformations of Stochastic Processes. P. I. Kuznetsov, R. L. Stratonovich, and V. I. Tikhonov, Eds, Oxford: Pergamon, 1965, pp. 64-76.

[29] H. S. Wang and P. C. Chang, "On verifying the first-order Markovian assumption for a Rayleigh fading channel model," IEEE Trans. Vehic. Technol., vol. 45, pp. 353-357, 1996.

[30] A. Abdi, "Comments on "On verifying the first-order Markovian assumption for a Rayleigh fading channel model"," IEEE Trans. Vehic. Technol., vol. 48, p. 1739, 1999.

[31] A. J. Rainal, "First and second passage times of Rayleigh processes," IEEE Trans. Inform. Theory, vol. 33, pp. 419-425, 1987.

[32] H. Allen Barger, "Dynamic characteristics of a narrowband land mobile communication channel," IEEE Trans. Vehic. Technol., vol. 47, pp. 216-224, 1998.

[33] P. C. F. Eggers, C. Thommesen, and J. B. Andersen, "On spatial power gradients," IEEE Trans. Vehic. Technol., vol. 49, pp. 235-238, 2000.

[34] A. Abdi, "Zero crossing problem in stochastic processes; Theory and applications," M. S. Seminar Report, Dept. of Elec. and Comp. Eng., University of Tehran, Tehran, Iran, Feb. 1996 (in Persian). 

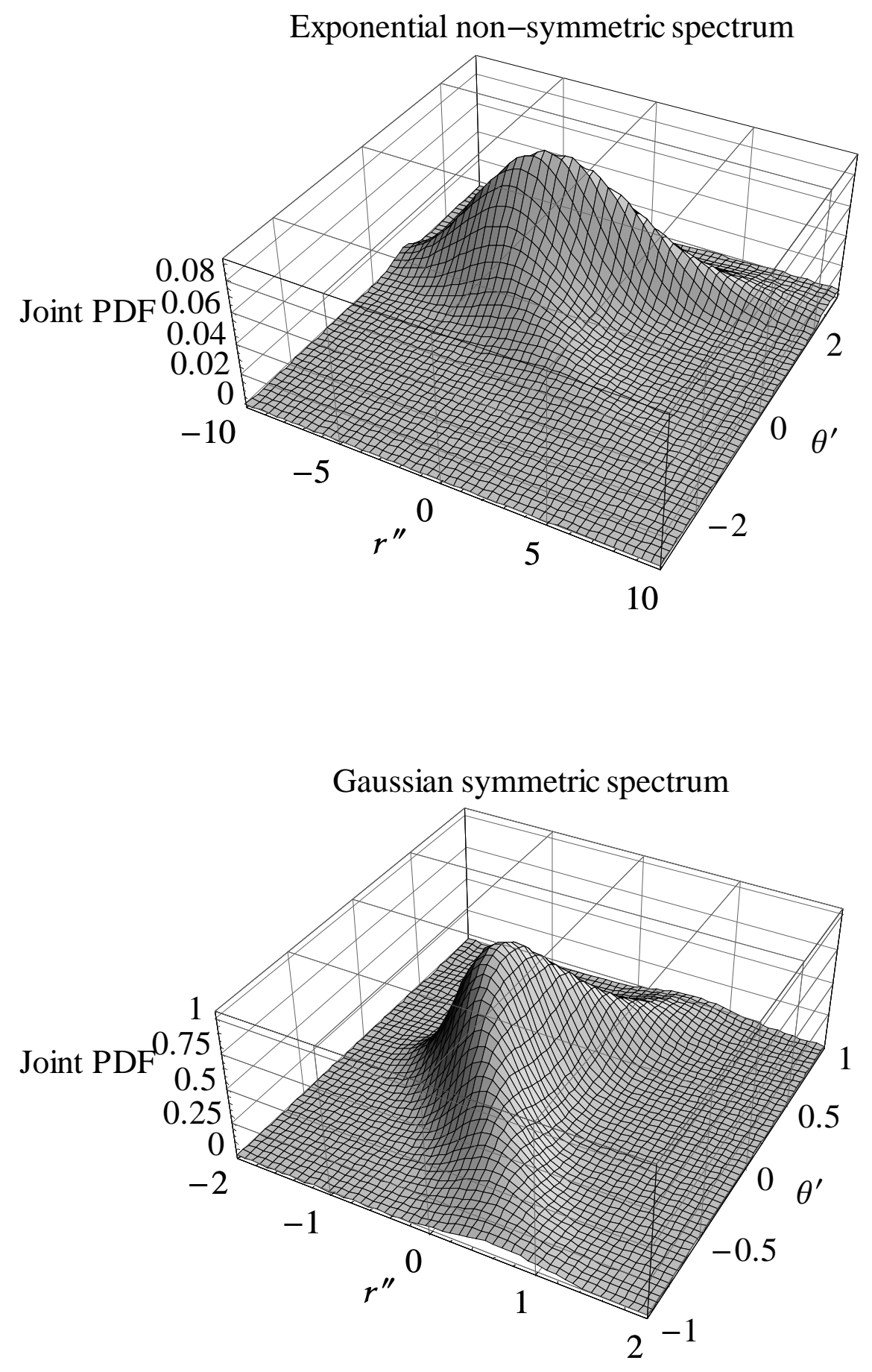

Fig. 1. The joint probability density function of $R^{\prime \prime}$ and $\Theta^{\prime}$ for two different power spectra: Upper: Exponential non-symmetric spectrum $w_{I}(f)=\exp \left(f-f_{m}\right), \quad-2<f-f_{m}<2$, Lower: Gaussian symmetric spectrum $w_{I}(f)=\exp \left[-\left(f-f_{m}\right)^{2}\right], \quad-1<f-f_{m}<1$. 

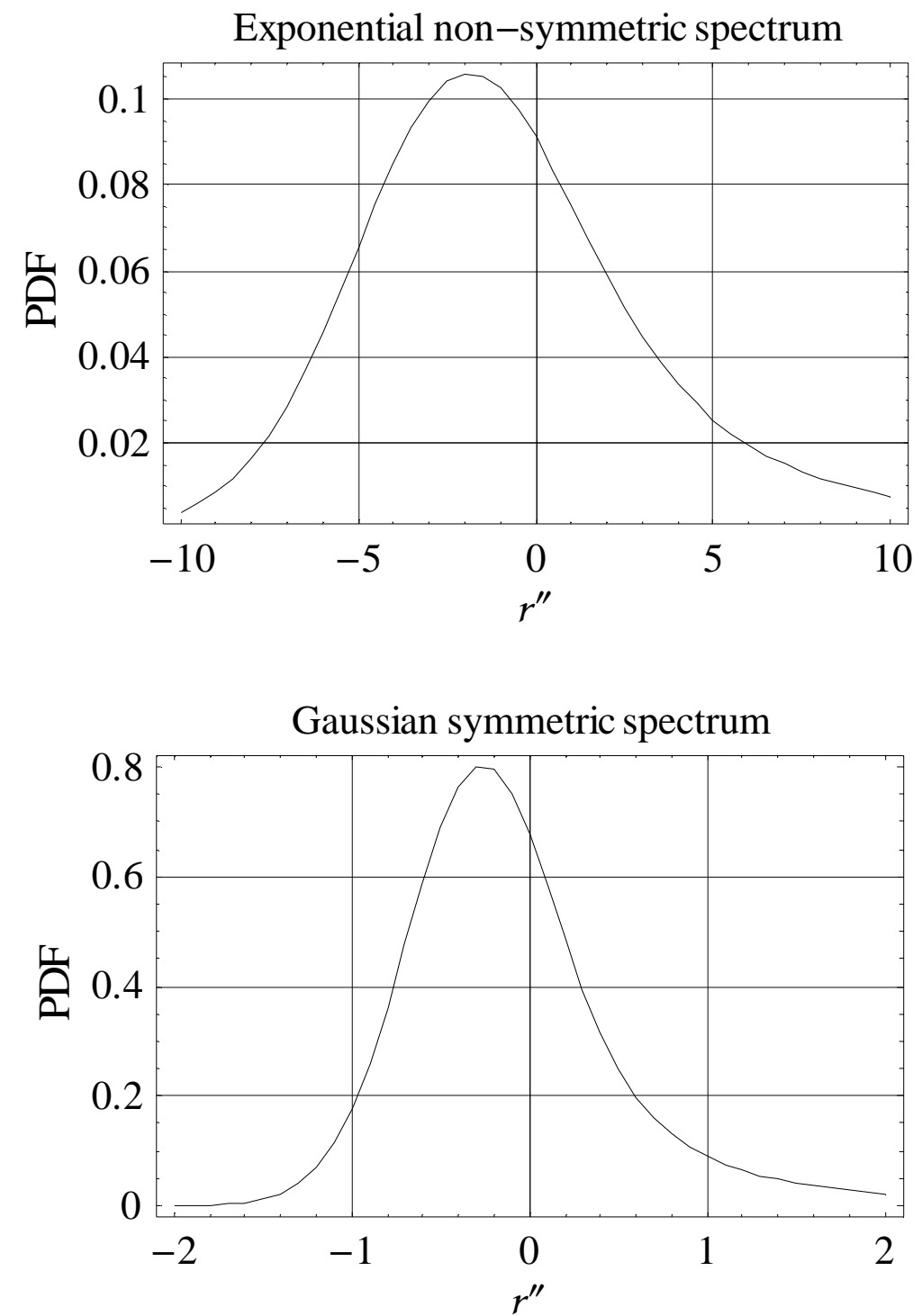

Fig. 2. The probability density function of $R^{\prime \prime}$ for two different power spectra:

Upper: Exponential non-symmetric spectrum $w_{I}(f)=\exp \left(f-f_{m}\right),-2<f-f_{m}<2$,

Lower: Gaussian symmetric spectrum $w_{I}(f)=\exp \left[-\left(f-f_{m}\right)^{2}\right],-1<f-f_{m}<1$. 


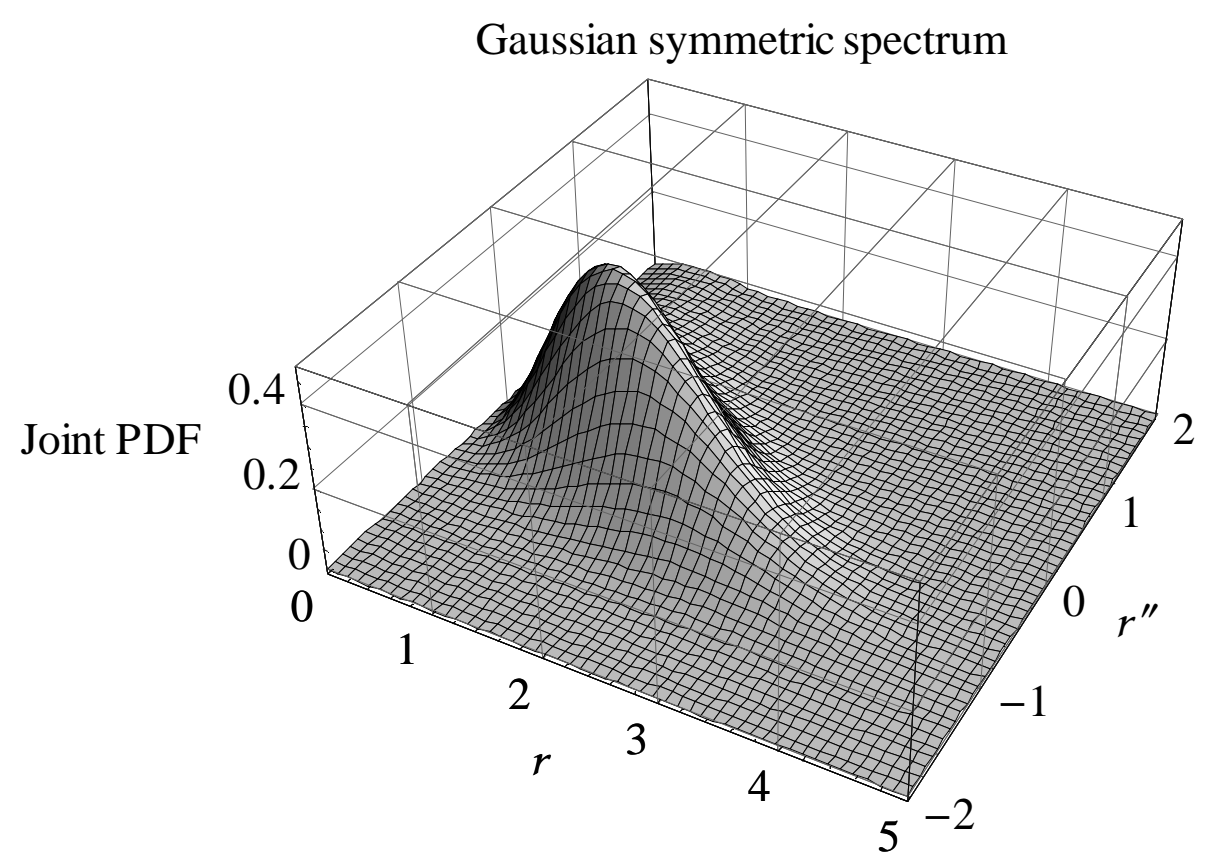

Fig. 3. The joint probability density function of $R$ and $R^{\prime \prime}$ for the Gaussian symmetric spectrum $w_{I}(f)=\exp \left[-\left(f-f_{m}\right)^{2}\right], \quad-1<f-f_{m}<1$. 